\title{
Distinct Hippocampal Time Cell Sequences Represent Odor Memories in Immobilized Rats
}

\author{
Christopher J. MacDonald, Stephen Carrow, Ryan Place, and Howard Eichenbaum \\ Department of Psychology and the Center for Memory and Brain, Boston University, Boston, Massachusetts 02215
}

Previous studies have revealed the existence of hippocampal "time cells," principal neurons in CA1 that fire at specific moments in temporally organized experiences. However, in all these studies, animals were in motion; and so, temporal modulation might be due, at least in part, to concurrent or planned movement through space or self-generated movement (path integration). Here the activity of hippocampal CA1 neurons was recorded in head-fixed and immobile rats while they remembered odor stimuli across a delay period. Many neurons selectively and reliably activated at brief moments during the delay, as confirmed by several analyses of temporal modulation, during a strong ongoing $\theta$ rhythm. Furthermore, each odor memory was represented by a temporally organized ensemble of time cells composed mostly of neurons that were unique to each memory and some that fired at the same or different moments among multiple memories. These results indicate that ongoing or intended movement through space is not necessary for temporal representations in the hippocampus, and highlight the potential role of time cells as a mechanism for representing the flow of time in distinct memories.

\section{Introduction}

Episodic memories are characterized by the temporal organization of events that compose unique experiences (Tulving, 1983). The hippocampus has long been considered important for episodic memory (Vargha-Khadem et al., 1997), but until recently, a general mechanism for temporal organization of memories in hippocampal neural networks had not been identified. However, there are now multiple reports of neural activity in the hippocampal CA1 region that signals the flow of time (Manns et al., 2007; Pastalkova et al., 2008; Gill et al., 2011; MacDonald et al., 2011; Naya and Suzuki, 2011; Kraus et al., 2013). Most striking are descriptions of hippocampal "time cells" that fire briefly in sequence during periods between salient events in behaving rats. In all recordings of time cells to date, the rats moved continuously during the empty delay periods (Pastalkova et al., 2008; Gill et al., 2011; MacDonald et al., 2011; Kraus et al., 2013), and systematic examination revealed that the temporal modulation of firing co-occurred with variation in firing rate associated with movement variables, including location, head direction, and speed (MacDonald et al., 2011). A more recent analysis of hippocampal neuronal firing patterns in animals running on a treadmill shows that time cells signal the distance traveled as well as time elapsed (Kraus et al., 2013). These results show that, although location and other movement-related variables

Received April 10, 2013; revised Aug. 1, 2013; accepted Aug. 7, 2013.

Author contributions: C.J.M. and H.E. designed research; C.J.M., S.C., and R.P. performed research; C.J.M. analyzed data; C.J.M. and H.E. wrote the paper.

This work was supported by National Institute of Mental Health Grant MH095297. We thank Dr. Yoshikazu Isomura for sharing his head-fixation protocol that we adapted and Dr. Matt Wachowiak for advice on related issues early on.

The authors declare no competing financial interests.

Correspondence should be addressed to Dr. Howard Eichenbaum, Department of Psychology, Center for Memory and Brain, 2 Cummington Mall, Boston, MA 02215. E-mail: hbe@bu.edu.

DOI:10.1523/JNEUROSCI.1537-13.2013

Copyright $\odot 2013$ the authors $\quad 0270-6474 / 13 / 3314607-10 \$ 15.00 / 0$ are not sufficient to account for the temporal signal (MacDonald et al., 2011), they are nevertheless typically encoded along with a representation of time. In addition, Pastalkova et al. (2008) reported that time cell sequences predict the spatial trajectory that is taken by a rat after a delay period, suggesting that time cell ensembles may be involved in planning a future path.

Consequently, it remained unclear whether movement-generated cues that could support path integration (McNaughton et al., 2006; Kraus et al., 2013) or the planning and/or execution of spatial trajectories after the delay underlie the hippocampal timing signal (Pastalkova et al., 2008; Gill et al., 2011; Kraus et al., 2013). Alternatively, hippocampal cells may represent the flow of time per se, and their development may not require ongoing movements or the planning and execution of distinct spatial trajectories. To address these issues, we eliminated movement variables by recording from head-fixed rats as they performed a simple delayed matching to sample task. This protocol allowed us to test three critical questions: Are time cells present in rats when they are immobile? If so, how do time cells represent specific odor memories? Finally, is the $\theta$ rhythm, which is observed in moving rats and commonly thought to organize spatial firing patterns (Buzsáki, 2005), present during memory performance even when movement is eliminated?

\section{Materials and Methods}

Subjects and behavior

Five male Long-Evans rats were water restricted and maintained at a weight of $400-450 \mathrm{~g}$. All protocols were approved by the Boston University Institutional Animal Care and Use Committee. The head-fixed apparatus and protocol was adapted from Isomura et al. (2009). Before training, a small headpiece was surgically cemented onto the rat's head. The aluminum headpiece was lightweight $(12 \mathrm{~g})$ and had two thin rails that ran along the left and right side of the rat's head. The rat's body rested inside an aluminum support tube that was $16.5 \mathrm{~cm}$ long and $7.75 \mathrm{~cm}$ in diameter $(0.635 \mathrm{~cm}$ thick wall $)$. However, the top half of the support tube could be slid up and down a thin aluminum wall $(0.25 \mathrm{~cm})$ mounted on 
each side of the tube so that rats with implanted hyperdrives could enter in the beginning of the recording session. The rat's head extended from one end of the tube and was fixed into position by gently sliding the rails into receptacles positioned to the left and right of the rat's head, then locked into place. Odor and lick spouts were positioned $\sim 2 \mathrm{~cm}$ in front of the rat's nose and mouth, respectively, through which odors and water were presented by operation of solenoid valves. A small photobeam detector was positioned in front of the lick spout to detect licks.

The memory testing protocol was a delayed matching to sample task wherein rats indicated whether a test odor "matched" or "mismatched" a preceding sample odor by licking at a water-spout (see Fig. 1). Each trial began with the presentation of a sample odor for $1 \mathrm{~s}$, followed by a constant delay period during which no odor was presented, then concluded with presentation of a test odor. For three rats (Rats 1-3), the test odor lasted $1 \mathrm{~s}$, followed by a 2 to $3 \mathrm{~s}$ response period during which the rat indicated a "match" or "mismatch" (Lu et al., 1993). For the other two rats (Rats 4 and 5), the test odor was presented for up to $2 \mathrm{~s}$ and licks during presentation of a matching test odor resulted in delivery of $\sim 50 \mu \mathrm{l}$ of water to the water-spout, whereas licks during presentation of a mismatching odor were not rewarded. For Rats $1-3$, an incorrect lick during the response period of mismatch trials ("false alarms") resulted in the start of the intertrial interval (ITI) that was signaled by a house-light. Rats 4 and 5 were required to emit 5-12 licks during presentation of the test odor to score a hit; the exact criterion lick count was specific to each rat, and the rat had to emit the same criterion to score a false alarm. This strategy was undertaken to counter a bias toward licking on each trial in this set of rats. After a correct trial, the ITI was on average $15 \mathrm{~s}$ (range, $12-18 \mathrm{~s}$ ); and after incorrect trials, the ITI was $25 \mathrm{~s}$ (range, 22-28 s). Trials were aborted and the ITI began if the rat licked during the delay period. The "no-lick" penalty enforced during the sample odor and delay period was adopted in the earliest stages in training because we noticed that some rats moved their hind legs while licking. Because of this penalty, we found that the rats remained still during the sample and delay period. All but one rat did move their hind legs shortly after starting to lick in response to the test odor.

The delay was different for each rat and ranged from 2 to $5 \mathrm{~s}$. For four rats, the delay period was fixed throughout the recording session. For one rat (Rat 3), the length of the delay period varied randomly between 4 and $5 \mathrm{~s}$ ( $0.25 \mathrm{~s}$ increments) during two sessions and $4.5-5.5 \mathrm{~s}(0.25 \mathrm{~s}$ increments) in a third session because we found that variable delays facilitated learning and stable performance in this rat. For Rats 1-3, only two odors were used throughout the session, whereas four odors were used for Rats 4 and 5 . The sample and test odors were chosen randomly to equate the occurrence of match and mismatch trials throughout the session.

\section{Electrophysiology}

The rats were prepared for implantation of the hyperdrive when performance stabilized at $>70 \%$ correct on three consecutive sessions. Following a standard surgical protocol (MacDonald et al., 2011), a hyperdrive containing 24 tetrodes was implanted to target the left hemisphere of the rat's dorsal hippocampus ( $\mathrm{AP}-3.6 \mathrm{~mm}$; $\mathrm{ML}=2.8 \mathrm{~mm}$ ). Each tetrode consisted of four nichrome wires $(12.5 \mu \mathrm{m}$ diameter; California Fine Wire) gold plated to lower the impedance to $200 \mathrm{k} \Omega$ at $1 \mathrm{kHz}$. At the end of surgery, each tetrode was lowered $\sim 850 \mu \mathrm{m}$ into tissue. After $5-7 \mathrm{~d}$ of recovery, the tetrodes were lowered over $10-20 \mathrm{~d}$ toward the CA1 layer, using the progressive increase in $\theta$ amplitude, the appearance of sharpwave and ripple events, and finally $\theta$-modulated and complex-cell spiking to localize CA1 (Fox and Ranck, 1981; Buzsáki et al., 1983).

Once the tetrodes were placed in a desired location, the rats were tested for $2 \mathrm{~h}$ or until clear signs of satiation ( $>15$ trials with no licking). The electrical signal recorded from the tips of the tetrodes was referenced to a common skull screw over the cerebellum and differentially filtered for single unit activity $(154 \mathrm{~Hz}$ to $8.8 \mathrm{kHz})$ and local field potentials $(1.5-400$ $\mathrm{Hz}$ ). The amplified potentials from each wire were digitized at $40 \mathrm{kHz}$ and monitored with a Multineuron Acquisition Processor (Plexon). Action potentials from single neurons were isolated using time-amplitude window discrimination through Offline Sorter (Plexon). Individual pyramidal neurons were isolated by visualizing combinations of waveform features (square root of the power, spike-valley, valley, peak, principal components, and time-stamps) extracted from wires making up a single tetrode (i.e., "cluster cutting"). We used conventional methods to identify putative pyramidal neurons and distinguish them from interneurons based upon firing rates and waveforms (Csicsvari et al., 1999). All successive action potentials from isolated neurons had interspike intervals $>2 \mathrm{~ms}$.

\section{Analysis of local field potentials}

Analysis of local field potential (LFP) frequency as a function of time used the multitaper functions written for MATLAB (MathWorks) that are freely available as part of the Chronux toolbox (Mitra and Bokel, 2008; http://www.chronux.org). The trial-averaged multitapered time-frequency spectrum was determined (mtspecgram.m) using a window size of $1 \mathrm{~s}$ that started at the beginning of the sample odor and was slid across time using $100 \mathrm{~ms}$ increments until $1 \mathrm{~s}$ into the test odor. The multitapered spectrum was generated using mtspectrumc.m. For both analyses, the time-bandwidth product was 3 , and the number of tapers used was 5 . We used an ANOVA to test $(p<0.05)$ whether trial-averaged power during a trial differed depending on frequency (MacDonald et al., 2011). For this analysis, we used the CA1 tetrode with the largest frequency content between 4 and $12 \mathrm{~Hz}$ in each recording session.

\section{Statistical analysis of spike data}

The time stamps for sample and test odor onsets and offsets were recoded via a Med Associates interface (Med Associates) with the Multineuron Acquisition Processor (Plexon), and the spike trains obtained from single neurons were aligned to the onset of the sample odor or delay period depending on the analysis. In all analyses, unless otherwise stated, we used correct trials in which the neuron had at least one action potential during the delay period.

During the delay period, the LFP from each tetrode was filtered between 4 and $12 \mathrm{~Hz}$ using a Butterworth filter. This signal was converted to an instantaneous phase by first applying a Hilbert transformation, then the four-quadrant inverse tangent of the real and imaginary components, and finally shifting the phase so that $0^{\circ}(0$ radians) corresponded to the trough of local $\theta$.

Using this phase signal, the delay period was segmented into contiguous, nonoverlapping time bins such that the peaks of successive $\theta$ cycles demarcated bin boundaries. Because bins were determined by the $\theta$ rhythm in a trial, the width of the bins in real time was not constant within or across trials. This procedure was implemented for each tetrode separately. Next, on each trial, the spikes recorded from a neuron during the delay period were placed into the appropriate $\theta$-related time bin that was generated from the LFP sampled from the same tetrode and converted to a firing rate based upon the duration of the $\theta$ cycle. The firing rates from each trial were then smoothed using a Gaussian-kernel ( $\sigma=$ $1.5 \theta$ cycles). Finally, the data were separated according to the odor that started the trial to define different trial types.

With the data organized in this way, for each neuron we computed the trial-averaged firing rates across $\theta$ cycles during the delay period. Firing rates were computed in this way for each trial type separately. Because the number of $\theta$ cycles could vary from trial to trial, for this analysis and all subsequent ones, we only evaluated the firing rates for the first $\theta$ cycle in the delay to the last $\theta$ cycle that had at least six trials. This step ensured that $\theta$ cycles near the end of the delay had a sufficient number of samples to estimate the firing rate and excluded $<5 \%$ of the total $\theta$ cycles in all trials.

Next, we explored whether individual cells activated reliably during a specific period within the delay between sample and test odor presentations. A neuron was considered a "time cell" if all five of the following criteria for temporal modulation were met for the trials composing at least one sample-odor defined trial type:

Generalized linear model. The firing rates in each $\theta$ cycle during the delay were modeled as $\mathrm{X} \sim \operatorname{Poisson}(\lambda)$ (McCullagh and Nelder, 1989). The model was formulated as shown in Equation 1 and fit to data from each neuron and trial type separately. On the right hand side of the expression, the $\alpha$ term is a constant. The first summation operates on time-related predictors $(t)$ and their coefficients $\beta$, with $t$ being a dummy variable to indicate the $i$ th $\theta$ cycle during the delay period (for a similar implementation, see also Stapleton et al., 2006). 


$$
\ln \lambda_{t}=\alpha+\sum_{i=1}^{I} \beta_{i} t_{i}
$$

The generalized linear model was fit to the data using MATLAB (version R2012b, MathWorks; generalizedlinearmodel.fit). To test whether a neuron was temporally modulated under this framework, we compared the deviance of a model with time-dependent predictors ( $\theta$ cycles; Eq. 1$)$ and a constant (a time-independent predictor) to the deviance of a model that included only a constant. A model with more parameters than an alternative model will always have a smaller deviance (i.e., when the latter is nested in the former and both are compared with the saturated model) but is preferred only if the reduction in deviance is greater than what one would expect based upon expanding the number of parameters in the model. Thus, an analysis of deviance tested whether the reduction in deviance was greater than a $\chi^{2}$ random variable with degrees of freedom equal to the number of number of additional time-dependent predictors $(p<0.05)$ (McCullagh and Nelder, 1989).

ANOVA. We conducted a one-way ANOVA on firing rates across $\theta$ cycles. A neuron was considered temporally modulated for a trial type if there was a main effect of $\theta$ cycle $(p<0.05)$.

Nonparametric random-shift test. Here we tested whether the trial averaged $\theta$ cycle firing rates for a trial type were greater than expected if the spike trains were randomly shifted in time. We shifted a neuron's spike train on each trial by a random duration, and the spike train was wrapped around to the beginning of the delay when the shift brought it past the end of the delay. The shifted spikes were placed into the appropriate $\theta$-generated cycle, smoothed using a Gaussian kernel ( $\sigma=1.5 \theta$ cycles), and then we computed a trial average using the surrogate dataset. We repeated this process 999 more times to generate a null distribution of firing rates for each $\theta$ cycle. If the actual trial-averaged firing rate (also smoothed with a Gaussian kernel with $\sigma=1.5$ ) during at least one $\theta$ cycle was $>95 \%$ of the firing rates in the same $\theta$ cycle's null distribution, the cell was considered temporally modulated. This procedure largely preserves the temporal structure of the spike train on each trial but decouples it from the consistent timing of the putative firing field.

Correlation between subsets of trials. To assess reliability across trials, we computed the Pearson-product moment correlation between trialaveraged activity from even-numbered and odd-numbered trials for each odor trial type. A firing field was considered reliable if this correlation was significant $(p<0.05)$.

Identification of a firing field. To define a single time field, we located $\theta$ cycles for which activity exceeded the half-maximum firing rate. If the cycles identified in this way were separated in time by $\leq 2 \theta$ cycles, they were considered part of the same firing field. Otherwise, the cycles were considered part of different fields. The length of a single firing field was equal to the number of $\theta$ cycles of which it was that composed. Cells with single firing fields that were longer than two-thirds of the delay period were not considered time cells.

\section{Spike- $\theta$ phase relationship}

For each neuron and trial type, action potentials during the delay were assigned a phase based upon the locally derived $\theta$ rhythm. We computed circular means and SD using the Circular Statistics Toolbox (Berens, 2009) available for MATLAB (version R2012b, MathWorks). A Rayleigh test was conducted to test whether the distribution of spike phases was uniform $(p<0.05)$. We also tested whether the magnitude of phase locking was different between time cells and non-time cells by computing the average length of the resultant vector for each group.

\section{Temporal information content}

This measure was adapted from Skaggs et al. (1993) as follows:

$$
\text { temporal information content }=\sum_{i}^{N} P_{i}\left(\lambda_{i} / \hat{\lambda}\right) \log _{2}\left(\lambda_{\mathrm{i}} / \hat{\lambda}\right)
$$

Here, $N$ is equal to the number of $\theta$ cycles in the delay, $i$ indexes the $\theta$ cycle in the delay, $p_{\mathrm{i}}$ is equal to the probability for occupancy of $\theta$ cycle $i$, $\lambda_{i}$ is equal to the average firing rate in $\theta$ cycle $i$, and $\hat{\lambda}$ is equal to the overall mean firing rate.

\section{Neural ensemble analyses}

The goal of these analyses was to generate a metric for the similarity between temporal patterns of neural ensemble activity across paired conditions.

First we considered a "between-odor" analysis that quantifies the similarity of ensemble time cell firing patterns during the delay following different sample odors. For a given sample-odor-defined trial type, each cell's firing rate during the delay was characterized as a vector that was $t$ elements long, where $t$ indexed the $\theta$ cycle. Cells that were temporally modulated for one trial type were concatenated to generate a linearized population vector that was $n \times t$ elements long, where $n$ is the number of time cells, and we considered only ensembles that contained at least three cells. For the same time cell ensemble, linearized population vectors were generated using their activity in all the remaining trial types. The Pearson product moment correlation was computed by correlating the population vector composed of time cells from each trial type to a population vector made up of the same ensemble using activity from each of the remaining trial types. The "between-odor" correlation was the average of all of these values computed for time cells from each trial type. As an example, in the case where four sample odors were used, cells that were temporally modulated for odor 1 (see criteria above) were used to create population vectors for each of the four trial types. Then the population vector for the odor 1 trial type was cross-correlated with the population vector for the same ensemble for the odor 2, 3, and 4 trial types to yield three correlation values. This process was repeated for each odor (i.e., a population vector characterizing activity for cells that were temporally modulated for odor 2 was cross-correlated with the population vector the same ensemble following odors 1,3 , and 4, and so on). In this way, 12 population correlations ( 3 for each trial type) were obtained and the average of these values defined the "between-odor" correlation for the rat. We also report the number of correlations pooled across rats that were statistically significant.

As one control comparison, we generated a population vector for even-numbered correct trials and odd-numbered correct trials of each trial type and cross-correlated these to yield the ensemble correlation for each trial type. The average of these correlations across trial types is referred to as the "same-odor" correlation, and the total number of correlations across rats that were statistically significant is reported.

As a second control, we calculated the expected correlation values between independent population vectors (Leutgeb et al., 2004). Similar to the "between odor" analysis described above, cells were identified as temporally modulated for one trial type and their firing rate series were concatenated to generate an $n \times t$ linearized population vector composed of cells temporally modulated for that trial type. The population vector composed of the same time cells in a second trial type was also generated, but the ordering of cells was rearranged so that the firing rate series of each cell in the first population vector was randomly paired with that of a different cell in the second population vector. The Pearson product moment correlation was computed using these two linearized vectors. The pairings of cells were rearranged 1000 times, and the average correlation was recorded. Time cells identified for one trial type were compared with their rearranged activity in all remaining trial types, as in the "between odor" analysis. The "random" correlation was the average of all of these values computed for time cells from each trial type. This resampling approach estimates the expected correlation coefficient when the population vectors are explicitly rearranged to make activity observed in one condition uncorrelated with that in the other, with the assumption that the temporal firing patterns of different cells during the delay are uncorrelated.

We also explored whether time cell ensemble activity predicted accuracy of responses by examining population coding during the delay period for correct versus error trials for each trial type using a similar approach. We identified cells that were temporally modulated for a given odor trial type and cross-correlated the population vector for correct 
trials with that for error trials. Here, we added an additional constraint that a cell was only included in a population vector for a trial type if, in addition to it being temporally modulated for the trial type, there were at least six correct and six error trials. The average of these correlations obtained for each trial type defined the "correct-error" correlation, and the total number of statistically significant correlations across rats is reported. The control comparison for this analysis was the "same-odor" correlation described above. (The "same-odor" correlation used only correct trials.) We also compared the "correct-error" correlation to the "random" correlation described above. The "correct-error" analysis was also extended to characterize population coding during the sample odor period. The same computations were carried out as described above, but only cells that were temporally modulated during the sample odor period were included.

To determine whether ensemble activity patterns during the delay predicted the ensuing behavioral response, we investigated ensemble coding during the delay after each odor for correct "go" versus correct "no-go" trials. For cells that were temporally modulated for a given odor trial type, population vectors were constructed using trials that either ended in a "go" or "no-go" response for the trial type in question. The cross-correlation between the "go" and "no-go" population vector was computed in this way for each trial type and the average of these values defined the "go-no-go" correlation. The total number of comparisons that yielded statistically significant correlations across rats is also reported. For this analysis, the control condition was the "same-odor" correlation as computed above, and the "random" correlation was also used for a comparison.

Finally, we tested whether correlations in two different conditions (e.g., "between-odor" correlation vs "same-odor" correlation) were different through a paired sample $t$ test $(p<0.05)$. We also tested whether the "random" correlation differed from zero using a single sample $t$ test $(p<0.05)$.

\section{Results}

We recorded from CA1 neurons in rats performing a delayed matching to sample task using odors as memory cues (Fig. 1). Each trial began with the presentation of one from a set of 2 or 4 sample odors, followed by memory delay, and then presentation of a test odor; the memory delay was usually fixed for each rat but varied between 2 and $5 \mathrm{~s}$ for different rats. If the test odor was the same as the sample (a "match"), the rat could lick at a water-spout to obtain a reward. Trials on which the test odor differed from the sample ("mismatch") were not rewarded, and rats learned to withhold lick responses to "mismatch" test odors. Once performance stabilized at $\geq 70 \%$ correct across three training sessions, rats were implanted with multiple tetrodes targeting the CA1 pyramidal cell layer and recordings commenced during memory testing. Here, we focus on 11 38-205 trial recording sessions from five rats whose average performance was $79 \pm 4 \%$ (mean $\pm \mathrm{SE}$; range, 69-91\%) correct.

Using standard criteria for unit isolation in tetrode recordings, we identified a total of 833 putative CA1 pyramidal neurons. The average firing rate of identified neurons throughout the session was $0.86 \pm 0.05 \mathrm{~Hz}$ (see Materials and Methods). For the analyses described here, we eliminated the possibility of sampling from the same cells across multiple recording sessions by using data from each tetrode in only the recording session from which the most were neurons were isolated, and then pooled cells across sessions to compose one ensemble of neurons for each rat. The total number of cells from each rat used in further analyses was as follows: 58 (isolated across 2 sessions) from Rat 1, 46 (isolated across 3 sessions) from Rat 2, 31 (isolated in 1 session) from Rat 3, 92 (isolated across 4 sessions) from Rat 4, and 154 (isolated in 1 session) from Rat 5 . The average number of cells that composed each neural ensemble was $76.2 \pm 21.9$ (mean $\pm \mathrm{SE})$.

\section{The $\theta$ rhythm was prominent throughout trials, and many neurons fired at a preferred phase of ongoing $\theta$}

Local network oscillation in the $\theta$ range $(4-12 \mathrm{~Hz})$ is thought to be a functionally important rhythm for the spatial and memory representations coded by the hippocampus (Hasselmo et al., 2002; Buzsáki, 2005). To examine whether a prominent $\theta$ rhythm exists in head-fixed rats engaged in a memory task, we generated trial-averaged time-frequency spectrograms to evaluate frequency content $(1-50 \mathrm{~Hz})$ during presentation of the sample odor, the delay period, and the first second of the presentation of the test odor (see Materials and Methods). Figure $2 a$ shows the results of this analysis for a representative recording session, and the frequency spectrum for the same data is plotted in Figure $2 b$ (see also Fig. $2 c$ for $1 \mathrm{~s}$ sample of raw LFP recorded during the delay of a single trial). A one-way ANOVA conducted on these data confirmed a significant main effect of frequency for each recording session (all $p$ values $<0.01$ ). Together, these data confirm a prominent band of trial-averaged $\theta$ across all trial phases and a peak frequency of $7 \mathrm{~Hz}$.

It has long been known that CA1 pyramidal cells are modulated by the phase of ongoing $\theta$ (Buzsáki, 1983; Bland, 1986; Fox et al., 1986). Given the prominent $\theta$ rhythm during the delay period, we tested whether neural activity was phase-locked to the ongoing $\theta$ rhythm for each odor trial type separately (see Materials and Methods). The activity of $54.8 \%$ (209 of 381) of the neurons was phase modulated by ongoing $\theta$ in at least one of the trial types (Rayleigh test; $p<0.05$ ). Figure $2 d$ shows polar plots that illustrate the phase of spiking during the delay for four example neurons that fired preferably between $90^{\circ}$ and $270^{\circ}$. The distribution of preferred phases across neurons that showed phase-locked activity was not uniform ( $p=0.03$; Rayleigh test) but instead showed an average preferred phase of $131 \pm 11.7^{\circ}$ (mean direction $\pm \mathrm{SE}$; trough of $\theta=180^{\circ}$ ), which is consistent with reports of putative CA1 pyramidal cells preferably spiking near the troughs of locally derived $\theta$ in freely moving animals engaged in a variety of behavioral tasks (e.g., Csicsvari et al., 1999; Mizuseki et al., 2009).

\section{Hippocampal neurons are selectively activated at particular moments during the delay}

To determine whether neurons activated at specific times during the delay, we took advantage of prominent $\theta$ rhythm and phase 
a
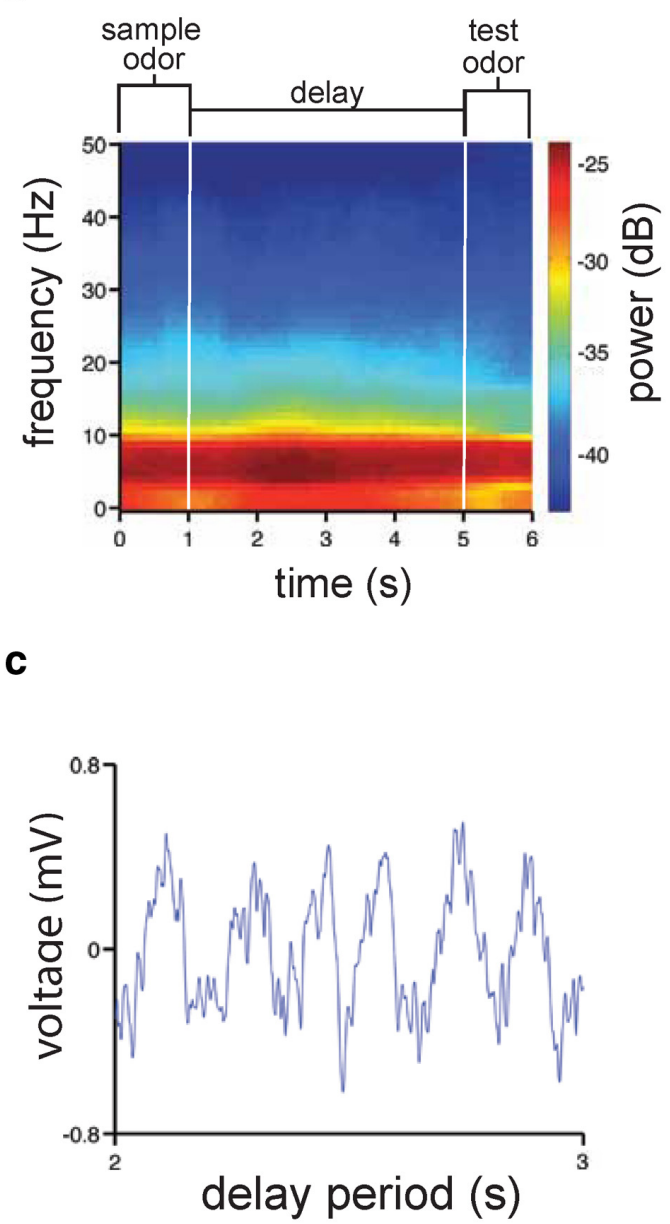

b

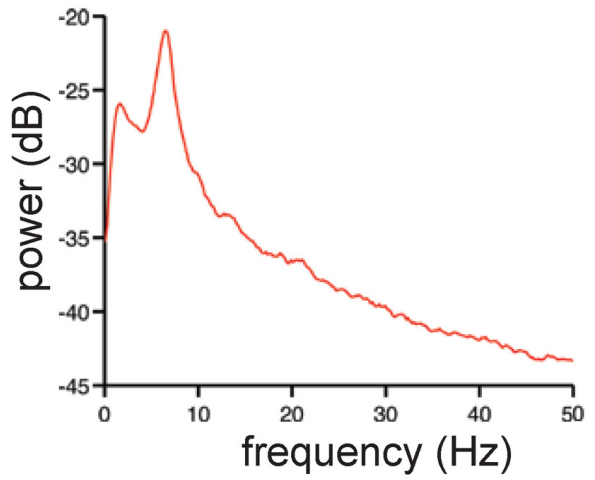

d
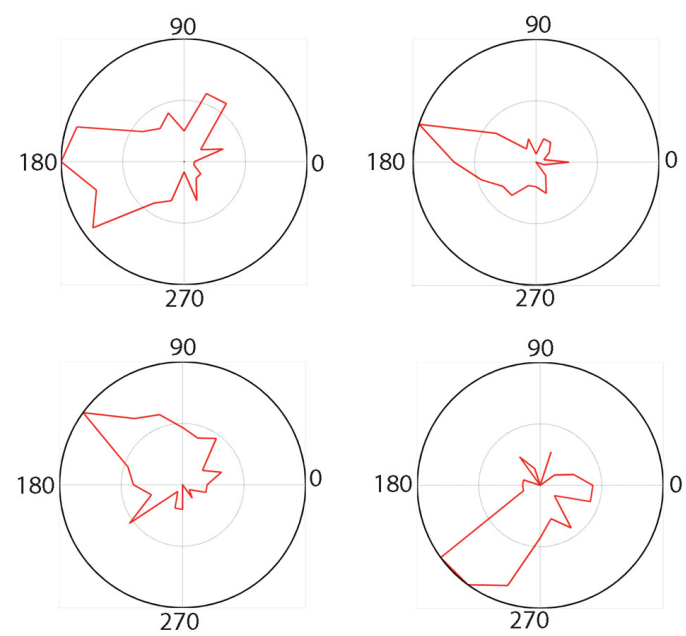

Figure 2. Throughout the trial, the LFP showed a prominent band of power within the $\theta$ range $(6-12 \mathrm{~Hz})$ and many neurons fired at a preferred phase of $\theta$. $\boldsymbol{a}$, The trial-averaged time-frequency spectrogram for sample, delay, and test periods for a representative recording session. $\boldsymbol{b}$, Multitapered frequency spectrum using all trials from the same recording session. $\boldsymbol{c}$, Representative LFP during $1 \mathrm{~s}$ of the delay period from the session illustrated above, and broad-band filtered between $1 \mathrm{and} 250 \mathrm{~Hz}$. $d$, Four polar plots illustrating normalized firing rates as a function of ongoing locally derived $\theta$.

modulation of most neurons. We divided the delay period into successive cycles of locally derived $\theta$ (e.g., Jezek et al., 2011) and evaluated firing rates as a function of $\theta$ cycle during the delay for each sample-odor defined trial type separately. Next, we explored whether there were cells that fired reliably during a particular period within the delay for at least one of the trial types, based upon meeting five statistical criteria.

First, we constructed a generalized linear model of firing rate during the delay period using a Poisson canonical link function (see Materials and Methods). The full model included timedependent predictors (theta cycle) and a time-independent predictor (constant). A reduced model that only included a constant was used to assess the significance of including theta cycle to predict firing rate during the delay. This analysis identified that the theta cycle predictor provided a significantly better fit of the data than a model using only a constant in $70.9 \%$ of the neurons for at least one trial type ( $p=0.05$; see Materials and Methods). Second, we used a one-way ANOVA to test whether firing rate varied across theta cycles $(p=0.05)$, and $54.9 \%$ of all of the neurons that fired during the delay met this criterion on at least one sample-odor defined trial type. In a third test, for each given trial type, we shifted the spike train during the delay period on each trial by a random duration to generate a distribution of trial averaged firing rates in each $\theta$ cycle based upon the null hypothesis that the observed spike trains were random in time. This analysis was used to test whether the observed trial averaged firing rate exceeded $95 \%$ of the null-distributed firing rates in any $\theta$ cycle for each trial type. A total of $89.7 \%$ of the neurons met this criterion in at least one $\theta$ cycle and one trial type. In a fourth analysis, for each neuron we correlated trial averaged activity in even-numbered and odd-numbered trials for each trial type, and $37.0 \%$ of the neurons were found to have a significant Pearson product moment correlation for at least one trial type $(p<0.05)$. Finally, we calculated the proportion of neurons that had distinct firing fields during the delay period for each trial type. A total of $58.3 \%$ neurons (222 of 381) had single firing fields during at least one of the trial types (Table 1 ).

We considered a neuron a time cell if a significant outcome was confirmed in the four statistical tests for trial-by-trial reliability and had a single time field. Applying this criterion (Table 1), $29.1 \%$ neurons of the neurons were time cells, and the majority $(85 \%)$ was temporally modulated for only one trial type. Moreover, Figure 3 shows the pattern of activity during the delay period (gray shaded area) for six representative time cells from each rat, using examples from different sample-odor defined trial types. Each time, the cell is selectively activated at a particular 
Table 1. Number and proportion (\%) of neurons exhibiting temporal modulation based upon different statistical analyses ${ }^{a}$

\begin{tabular}{|c|c|c|c|c|c|c|c|c|}
\hline Rat & No. of odors & Total cells & GLM & ANOVA & Shift & Even-odd & Time field & Time cells \\
\hline 1 & 2 & 58 & $30(51.7)$ & $22(37.9)$ & $46(79.3)$ & $15(25.6)$ & $28(48.3)$ & 11 (18.9) \\
\hline 2 & 2 & 46 & $30(65.2)$ & $20(43.5)$ & $43(93.5)$ & $13(28.3)$ & $21(45.7)$ & $11(23.9)$ \\
\hline 3 & 2 & 31 & $16(51.6)$ & $16(51.7)$ & $19(61.3)$ & $12(38.7)$ & $17(54.9)$ & $10(32.2)$ \\
\hline 4 & 4 & 92 & $69(75.0)$ & $50(54.3)$ & 84 (91.3) & $37(40.2)$ & $65(70.1)$ & $30(32.7)$ \\
\hline 5 & 4 & 154 & 125 (81.1) & 101 (65.6) & $150(97.4)$ & 64 (41.6) & 91 (59.1) & 49 (31.9) \\
\hline Total & 14 & 381 & $270(70.9)$ & $209(54.9)$ & 342 (89.7) & $141(37.0)$ & $222(58.3)$ & $111(29.1)$ \\
\hline
\end{tabular}

${ }^{a}$ For each subject, the number of sample odors used in testing and the number of isolated CA1 pyramidal cells are reported. In addition, the number of neurons (and percentage of the total) that exhibited reliable temporal modulation in at least one odor trial type is shown for each of several types of analyses described in the text conducted separately.

moment during the delay, and the entire delay period is represented by the combination of cells.

The average peak firing rate of a time cell was $2.72 \pm 0.14 \mathrm{~Hz}$ (mean $\pm \mathrm{SE}$ ). The ratio of infield to outfield firing rate during the delay period was $3.89 \pm 0.67$ (median \pm SE) and the average number of cycles in a firing field was $8.39 \pm 0.41$ (mean $\pm \mathrm{SE}$ ). The width of the firing field was significantly correlated with the time of peak firing rate, such that the firing duration increased later in the delay $(r=0.22, p=0.01)$, consistent with observations on time cells in the freely moving rat (MacDonald et al., 2011; Kraus et al., 2013). There was no difference between the proportion of time cells (56\%; or 62 of 111 ) and non-time cells ( $52 \%$; or 147 of 278 ) that were phase-locked to ongoing $\theta$ rhythm $\left(\chi^{2}=0.04, p=0.84 ; \chi^{2}\right.$ test $)$. However, the magnitude of $\theta$ modulation was greater $(Z=2.39, p=0.01$; Mann-Whitney $\mathrm{U}$ test) in time cells that were phase-locked (average resultant vector length $=0.31 \pm 0.01$; mean $\pm \mathrm{SE}$ ) compared with non-time cells that were phase-locked (average resultant vector length $=0.27 \pm 0.01$; mean $\pm \mathrm{SE}$ ).

To provide a qualitative comparison to previous reports of spatial information coded by hippocampal CA1 pyramidal cells, we computed the average temporal information content for time cells during the delay, adapting a conventional measure of spatial information content (Skaggs et al., 1993). The temporal information content was $0.44 \pm 0.02 \mathrm{bits} / \mathrm{spike}$ (mean $\pm \mathrm{SE}$ ), which is lower than the spatial information content reported for place cells recorded from freely moving animals in a familiar environment (typically 0.75-1.5 bits/spike) (Markus et al., 1995; Chen et al., 2013) but is comparable to scores reported in head fixed mice running on a virtual linear track devoid of prominent visual landmarks (0.35-0.40 bits/spike) (Chen et al., 2013). Temporal information scores cannot be compared quantitatively between these studies because the calculation depends heavily on how the data are binned, which varies among the studies. Nevertheless, we include these numbers for illustrative purposes. Together, these findings reveal that, even in rats that are immobile, hippocampal cells encode successive moments during an extended interval between a sequence of salient events during memory performance.

\section{Each odor memory is represented by a largely distinct pattern of time cell activity}

Next, we asked whether time cells have distinctive ensemble firing patterns during the delay between trial types that begin with different sample odors. Figure $4 a$ shows the temporal firing patterns of 49 time cells across four trial types recorded from Rat 5 in a single recording session. Each panel shows normalized firing rates for identified time cells over the course of the delay period after a particular sample odor presentation, and these ensemble firing rate plots are sorted by successive peak firing cycles. Comparisons of the identifications of neurons (see cell numbers on vertical axis) that are activated and associated with each trial type highlight the distinct ensemble representation of the delay period for each trial type.

To quantitatively measure the extent to which population firing patterns distinguished trial types that began with different sample odors, for each rat we composed population vectors of neural ensemble activity during the delay for each trial type (e.g., Fig. $4 a$, panels) and then correlated each of those ensemble patterns against population vectors constructed from the identical set of cells in the same ordering for each of the other trial types to obtain a set of "between-odor" correlations for each rat. For example, in the analysis of data from Rat 5, the population vector composed of 9 cells for trials that began with sample odor 1 (Fig. $4 a$ ) was compared with population vectors composed of the same 9 cells in the same order for trials that began with odor 2, with odor 3, and with odor 4. To compare firing patterns between trials that began with sample odor 2 with those for trials that began with other sample odors, similar correlations were performed for the 10 cell ensemble identified for trials that began with odor 2 against ensembles of the same 10 cells in the same order for the trials that began with odors 1, 3, and 4, and so on. These correlation coefficients were then averaged for each rat, and we also report the number of total number of correlation coefficients that were statistically significant pooled across all rats. As one control, we compared average "between-odor" correlation coefficients with average correlations between population vectors for even-numbered and odd-numbered trials that began with the same sample odor for each of the trial types ("same-odor" correlation; see Materials and Methods). As a baseline for the expected value of a correlation between randomized population vectors ("random" correlation), we used a resampling approach to compute the average correlation between independent population vectors, wherein each neuron in one vector is randomly paired with a neuron in the other vector (compare with Leutgeb et al., 2004).

The average population correlation for the "same-odor" control condition was $0.56 \pm 0.06$ (mean \pm SE; range, $0.38-0.75$; Fig. $4 b$ ), and 14 of 14 individual correlations were significant (range of $p$ values $\left.=1.7 \times 10^{-17}-8.9 \times 10^{-7}\right)$. The average correlation for the "between-odor" population vector correlations was $0.34 \pm 0.09$ (mean $\pm \mathrm{SE}$; range, -0.14 to 0.67 ; Fig. $4 b$ ), and 26 of 30 comparisons resulted in a significant correlation (range of $p$ values $\left.=1.6 \times 10^{-20}-0.39\right)$. The average correlation coefficient was significantly larger in "same-odor" than "between-odor" comparisons $\left(t_{(8)}=6.65, p=0.003\right.$; paired $t$ test), indicating that time cell ensemble representations following the same sample odor were more similar than those between different odors. In addition, the correlation coefficients for the "same-odor" and "between-odor" conditions were both significantly above the "random" correlation of $0.14 \pm 0.07$ (mean \pm $\mathrm{SE}$; both $p$ values $<0.02$; $t$ test), and the "random" correlation did not differ from zero $\left(t_{(4)}=1.97 ; p=0.12\right.$; one sample $t$ test). 

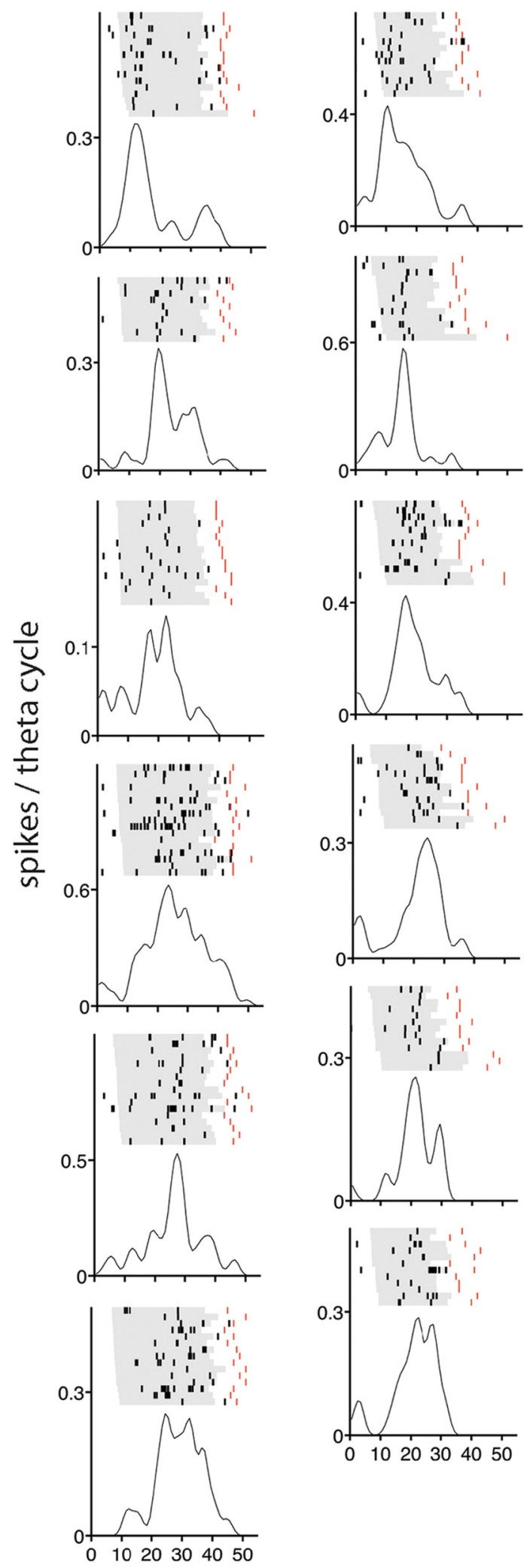
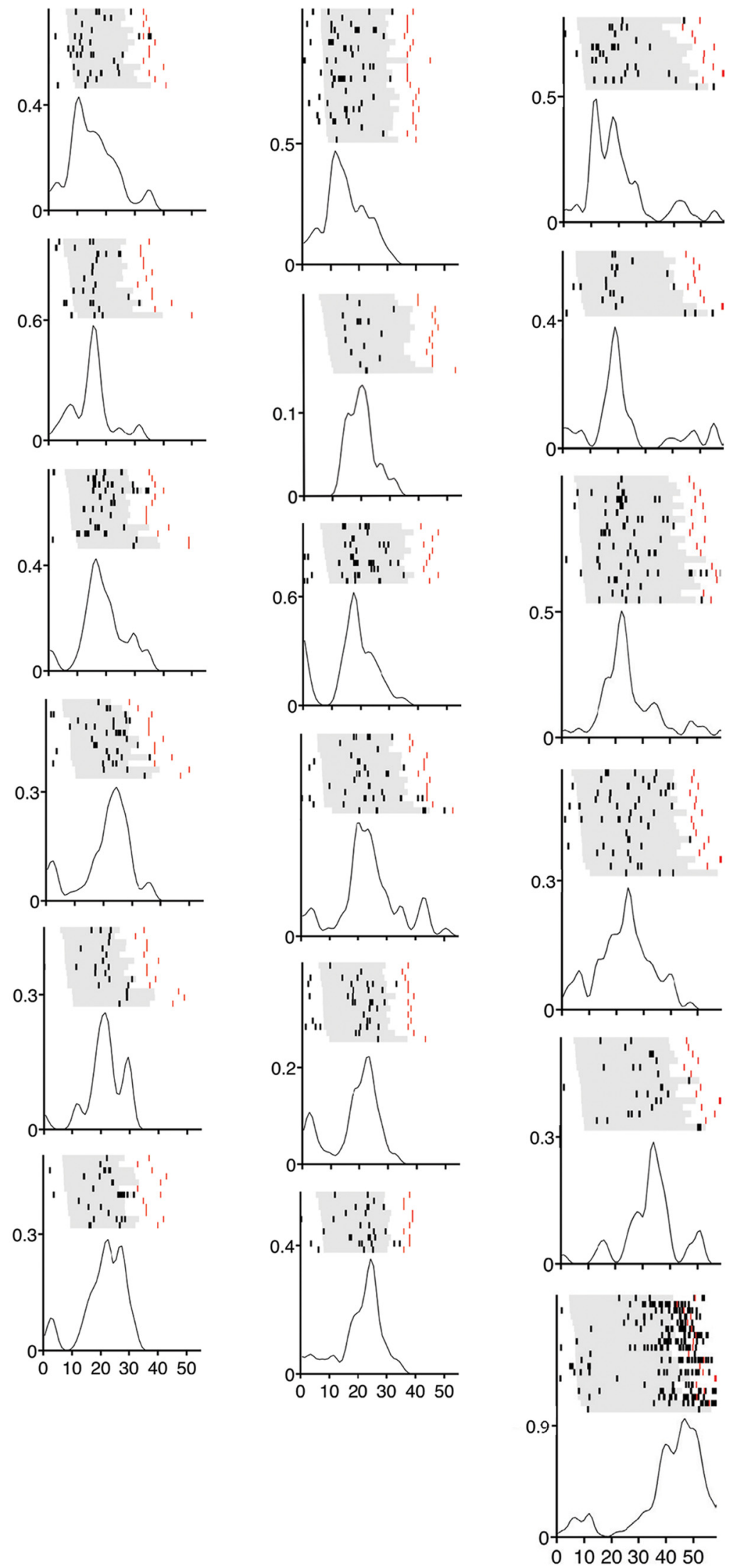

\section{theta cycle}

Figure 3. Hippocampal neurons fire at brief moments during the memory delay period. Each column depicts a raster plot and peristimulus time histogram showing neural activity from six time cells from each rat as a function of $\theta$ cycle. The gray area in the raster plot represents the memory delay period; the sample odor was presented during the $1 \mathrm{~s}$ preceding the delay and the test odor turned on when the memory delay ended. The red ticks indicate $1 \mathrm{~s}$ after the onset of the test odor. 
a

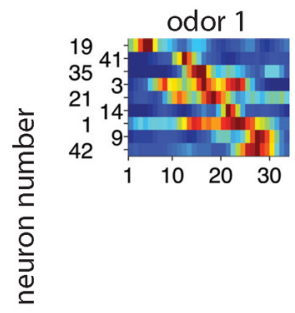

odor 2

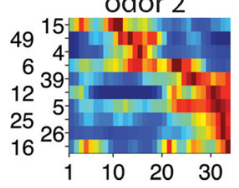

odor 3

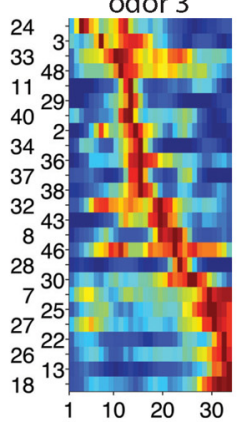

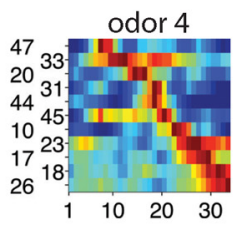

theta cycle

b

C 口 "random"

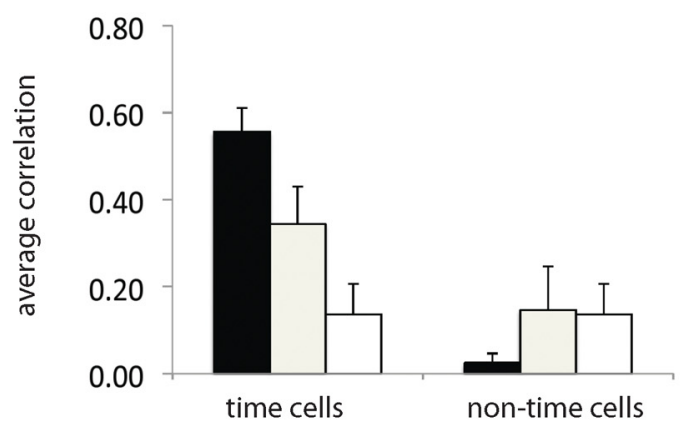

घ "same-odor" control $\square$ "correct vs. error" trials 口 "random"

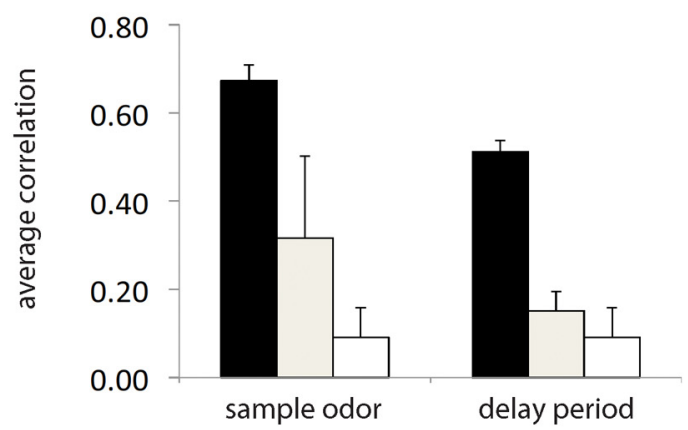

Figure 4. Odor memory representations during the delay for each sample odor defined trial type involved largely distinct, temporally organized neural ensemble activity. $\boldsymbol{a}$, Normalized firing rates over the delay for time cells (numerically labeled) for each of four sample-odor defined trial types in Rat 5 . $\boldsymbol{b}$, Average correlation coefficient between ensemble vectors for each trial type against the population vector for the same set of neurons in all other trial types ("between-odor"). As one control, the average correlation coefficient between subsets of trials (even vs odd) that began with the same odors is shown ("same-odor" control). As a second control, the average correlation coefficient between independent, randomly rearranged population vectors is shown ("random"). c, For ensembles of cells that were temporally modulated in the sample odor or delay period, shown is the average correlation coefficient correlation between population vectors from correct trials that began with the same odor and error trials that began with the same odor ("correct vs error" trials). The average correlation for the "same-odor" and "random" conditions are also shown.

These results indicate some degree of similarity between time cell ensemble firing patterns that follow different sample odors.

We also explored whether time cell population activity patterns predict the pending behavioral response. To examine this possibility, we correlated the ensemble firing patterns during the delay after each odor for trials in which the rat emitted a correct "go" response (licking) and for trials beginning with the same odor but ending in a correct "no-go" response (no licking). The average correlation for this "go-no-go" comparison was $0.49 \pm$ 0.03 (mean \pm SE; range, $0.21-0.75$ ), and 14 of 14 comparisons resulted in a significant correlation (range of $p$ values $=6.7 \times$ $\left.10^{-17}-0.03\right)$. This value was not different from the population correlation from the control condition $\left(t_{(4)}=1.38, p=0.24\right.$; paired $t$ test), and both of these values were different from the "random" correlation. This finding suggests that time cell ensembles do not incorporate information about the eventual behavioral response during the delay period, which is consistent with the task demand to compare the test odor to the sample before deciding on which response to perform.

As a final comparison, we conducted the analysis of "sameodor" versus "between-odor" population correlations using neurons that were not time cells (Fig. $4 b$ ). The average population correlation for the "same-odor" condition was $0.02 \pm 0.02$ (mean \pm SE; range, -0.03 to 0.1 ; Fig. $4 b$ ) and 4 of 14 individual correlations were significant (range of $p$ values $=1.1 \times 10^{-8}$ 0.72). The average correlation for the "between-odor" population vector correlations was $0.15 \pm 0.10$ (mean $\pm \mathrm{SE}$; range,
$0-0.52$ ), and 9 of 15 comparisons resulted in a significant correlation (range of $p$ values $=5.0 \times 10^{-24}-0.92$ ). The "same-odor" and "between-odor" population correlations for non-time cells were not different from the "random" correlation of $0.14 \pm 0.07$ (mean $\pm \mathrm{SE}$; both $p$ values $>0.13$; $t$ test), nor were they different from one another $\left(t_{(8)}=1.40 ; p=0.23\right.$; paired $t$ test). Moreover, both the "same-odor" and the "between-odor" population correlations for time cells were higher than those for the non-time cells (all $p$ values $<0.03$; paired $t$ test).

Together, these results indicate that odor memories are represented by distinct, temporally organized patterns of hippocampal neural activity, although some degree of commonalities in temporal structure is preserved across trial types that use different odors.

\section{Time cell representations predict accurate odor memories}

Do time cell sequences correspond to accurately encoded memories? To address this question, we correlated population vectors of time cell activity for correct trials against those for the same set of cells in the same order for error trials that began with the same odor (Fig. 4c). This analysis excluded one rat because there were an insufficient number of error trials to analyze (performance $=$ $91 \%$ correct), and we used the correlation between ensemble patterns from even-numbered and odd-numbered correct trials of the same sample-odor trial type as a correct "same-odor" control. The average population correlation in the "same-odor" condition was $0.51 \pm 0.03$ (mean \pm SE; range, 0.47-0.67), and all 9 
correlations were statistically significant (range of $p$ values $=$ $\left.1.1 \times 10^{-59}-8.9 \times 10^{-7}\right)$. The average correlation between time cell ensemble activity in correct versus error trials from the same trial type was $0.15 \pm 0.04$ (mean $\pm \mathrm{SE}$; range, $0.04-0.23$ ), and the number of comparisons that had a significant correlation was 5 of $8\left(\right.$ range of $p$ values $\left.=2.3 \times 10^{-7}-0.73\right)$. The difference between the average correlation coefficients in correct "same-odor" versus "correct-error" comparisons was significant $\left(t_{(3)}=6.25, p=\right.$ 0.008 ; paired $t$ test). Moreover, the average correlation coefficient between correct and error trials was not different from the "random" correlation $\left(t_{(3)}=0.60, p=0.58 ; t\right.$ test $)$, suggesting that the pattern of time cell ensemble activity in error trials was comparable with what would be expected in randomized neural populations.

Because population activity from error trials was poorly correlated with that of correct trials during the delay period, we tested whether the poor coding occurs during the sample odor presentation and thus might simply propagate through the delay. To address this possibility, we first identified cells that fired reliably during the sample odor period on correct trials using the same analysis as used for the delay period. This analysis revealed that $15 \%$ of the total population of cells ( 58 of 381) activated for at least one of the sample odors. Then we conducted a "correcterror" trial population analysis using these cells (Fig. 4c). The average "same-odor" population correlation was $0.67 \pm 0.03$ (mean \pm SE; range, $0.60-0.74$ ), and all four correlations were statistically significant (range of $p$ values $=8.06 \times 10^{-14}-4.4 \times$ $\left.10^{-3}\right)$. The average "correct-error" correlation was $0.32 \pm 0.19$ (mean \pm SE; range, -0.23 to 0.57 ), and only one correlation was statistically significant (range of $p$ values, 0.003-0.43). Unlike neural ensembles active during the delay period, there was no difference between the average population correlation in the correct "same-odor" and "between-odor" condition $\left(t_{(3)}=2.20\right.$ $p=0.12$; paired $t$ test), although all four correlations in the "between-odor" condition were lower by at least 0.12 . In addition, whereas the "same-odor" correlation coefficient was different from the "random" correlation $\left(t_{(3)}=6.00, p=0.009 ; t\right.$ test), the "correct-error" correlation was not $\left(t_{(3)}=1.10, p=0.35 ; t\right.$ test).

Collectively, the "correct-error" analyses indicate that time cell population activity patterns that predict the accuracy of subsequent memory-related judgments may be initiated during the odor coding, but ensemble activity during the delay more strongly predicts subsequent accuracy.

\section{Discussion}

The present results provide compelling evidence that hippocampal neurons encode successive brief moments that compose the flow of time within a distinct memory, even when location and head direction are fixed and movement is eliminated. These findings join previous reports of time cells recorded in rats and monkeys performing various memory tasks (Manns et al., 2007; Pastalkova et al., 2008; Gill et al., 2011; MacDonald et al., 2011; Naya and Suzuki, 2011; Kraus et al., 2013), thus expanding the generality of temporal coding of memories in the hippocampus. Furthermore, because the animals are motionless during and after each trial, the present results run contrary to the suggestion that time cells reflect idiothetic cues associated with movement or that they represent intended paths through space (Pastalkova et al., 2008). The present findings also indicate that hippocampal neurons can represent the flow of time outside of the integration of paths through space (McNaughton et al., 2006) and are consistent with the observation that hippocampal firing sequences occur before and therefore can arise without experiencing spatial trajectories (Dragoi and Tonegawa, 2011).

The peak firing rates of time cells observed here were lower than those reported in place cells (typically $\geq 10 \mathrm{~Hz}$ ), as well as in time cells in freely moving rats performing other memory tasks (Pastalkova et al., 2008; MacDonald et al., 2011). Higher peak firing rates of CA1 pyramidal cells have also been observed and associated with the presentation of salient stimuli in rats when they are relatively immobile (Wood et al., 1999; Moita et al., 2003; Itskov et al., 2011; MacDonald et al., 2011) and before movements when still (Lenck-Santini et al., 2008), and in head-fixed mice running in place (Harvey et al., 2009; Royer et al., 2012). However, CAl firing rates are reduced when rats move through space without walking (Foster et al., 1989; Terrazas et al., 2005; but see Song et al., 2005). Moreover, firing patterns of CA1 neurons typically involve only a few spikes per event during relative immobility when memories are "replayed" (Carr et al., 2011) or "preplayed" (Dragoi and Tonegawa, 2011), suggesting that high firing rates in single neurons are not necessary to convey information to downstream targets. These findings suggest that the relatively low peak CA1 firing rates observed here may reflect internal mechanisms that underlie the timing signal without the addition of external sensory or self-motion generated inputs. The magnitude of this timing signal may be approximately equivalent to that of the temporal modulation we previously extracted from firing patterns that was independent of the rat's location, behaviors, and their interactions (MacDonald et al., 2011).

The differences in temporal firing patterns of CA1 neurons observed here may be characterized as predominantly reflecting qualitative differences in temporal firing patterns ("global remapping") among different memories, and these ensemble firing patterns predict accurate memories for the odors. A minority of cells was common to ensembles that represented multiple odor memories, although rarely for all four trial types, and some of these neurons varied in their temporal patterns or firing rates ("rate mapping") for different odor memories, resulting in some commonalities among specific memory representations. Notably, Leutgeb et al. (2005) similarly observed a mixture of global and rate remapping of CA1 neurons in the spatial representations of different local environments. Alternatively, rate remapping in CA1 neurons was predominant in a recent study of rats performing a cued spatial maze task (Allen et al., 2012), as well as in earlier studies that have observed hippocampal cells firing at different rates as rats traverse the same places predicting spatial choices in a T-maze (e.g., Wood et al., 2000; Robitsek et al., 2013). One possible explanation of the different findings in the current study compared with the previous maze studies is that, because of the relatively low peak firing rates observed in the head fixed condition, some cells with suppressed firing rates would appear to "drop out" of the representation for some trial types; and conversely, other cells might produce detectable peak rates only in those same trial types. To the extent such a phenomenon is prevalent, global and rate remapping are indistinguishable. Regardless of this conundrum of interpretation, the present findings indicate that the hippocampus captures distinctions among memories by largely separate neural ensembles, whereas the commonalities in temporal structure among related memories are reflected in the consistently timed activation of relatively few neurons firing at the same or different rates across distinct memories. Therefore, the unique, temporally organized firing patterns for each odor-specific trial type observed here provide a potential mechanism for a representation of the flow of events and time in 
distinct memories as well as commonalities among the temporal structure of memories.

\section{References}

Allen K, Rawlins JN, Bannerman DM, Csicsvari J (2012) Hippocampal place cells can encode multiple trial-dependent features through rate remapping. J Neurosci 32:14752-14766. CrossRef Medline

Berens P (2009) CircStat: a Matlab toolbox for circular statistics. J Statistical Software 31:Issue 10.

Bland BH (1986) The physiology and pharmacology of hippocampal formation $\theta$ rhythms. Prog Neurobiol 26:1-54. CrossRef Medline

Buzsáki G (2005) Theta rhythm of navigation: link between path integration and lang mark navigation, episodic and semantic memory. Hippocampus 15:827-840. CrossRef Medline

Buzsáki G, Leung LW, Vanderwolf CH (1983) Cellular bases of hippocampal EEG in the behaving rat. Brain Res 287:139-171. Medline

Carr MF, Jadhav SP, Frank LM (2011) Hippocampal replay in the awake state: a potential substrate for memory consolidation and retrieval. Nat Neurosci 14:147-153. CrossRef Medline

Chen G, King JA, Burgess N, O'Keefe J (2013) How vision and movement combine in the hippocampal place code. Proc Natl Acad Sci U S A 110: 378-383. CrossRef Medline

Csicsvari J, Hirase H, Czurkó A, Mamiya A, Buzsáki G (1999) Oscillatory coupling of hippocampal pyramidal cells and interneurons in the behaving rat. J Neurosci 19:274-287. Medline

Dragoi G, Tonegawa S (2011) Preplay of future place cell sequences by hippocampal cellular assemblies. Nature 469:397-401. CrossRef Medline

Foster TC, Castro CA, McNaughton BL (1989) Spatial selectivity of rat hippocampal neurons: dependence on preparedness for movement. Science 244:1580-1582. CrossRef Medline

Fox SE, Ranck JB Jr (1981) Electrophysiological characteristics of hippocampal complex-spiking and $\theta$ cells. Exp Brain Res 41:399-410. Medline

Fox SE, Wolfson S, Ranck JB Jr (1986) Hippocampal theta rhythm and the firing of neurons in walking and urethane anesthetized rats. Exp Brain Res 62:495-508. Medline

Gill PR, Mizumori SJ, Smith DM (2011) Hippocampal episode fields develop with learning. Hippocampus 21:1240-1249. CrossRef Medline

Harvey CD, Collman F, Dombeck DA, Tank DW (2009) Intracellular dynamics of hippocampal place cells during virtual navigation. Nature 461: 941-946. CrossRef Medline

Hasselmo ME, Bodelón C, Wyble BP (2002) A proposed function for hippocampal $\theta$ rhythm: separate phases of encoding and retrieval enhance reversal of prior learning. Neural Computation 14:793-817. CrossRef Medline

Isomura Y, Harukuni R, Takekawa T, Aizawa H, Fukai T (2009) Microcircuitry coordination of cortical motor information in self-initiation of voluntary movements. Nat Neurosci 12:1586-1593. CrossRef Medline

Itskov PM, Vinnik E, Diamond ME (2011) Hippocampal representation of touch-guided behavior in rats: persistent and independent traces of stimulus and reward location. PLoS One 6:e16462. CrossRef Medline

Jezek K, Henriksen EJ, Treves A, Moser EI, Moser MB (2011) $\theta$-paced flickering between place-cell maps in the hippocampus. Nature 478:246-249. CrossRef Medline

Kraus BJ, Robinson RJ 2nd, White JA, Eichenbaum H, Hasselmo ME (2013) Hippocampal "time cells": time versus path integration. Neuron 78:1-12. CrossRef Medline

Lenck-Santini PP, Fenton AA, Muller RU (2008) Discharge properties of hippocampal neurons during performance of a jump avoidance task. J Neurosci 28:6773-6786. CrossRef Medline

Leutgeb S, Leutgeb JK, Treves A, Moser MB, Moser EI (2004) Distinct ensemble codes in hippocampal areas CA3 and CA1. Science 305: 1295-1298. CrossRef Medline
Leutgeb S, Leutgeb JK, Barnes CA, Moser EI, McNaughton BL, Moser MB (2005) Independent codes for spatial and episodic memory in hippocampal neuronal ensembles. Science 309:619-623. CrossRef Medline

Lu XC, Slotnick BM, Silberberg AM (1993) Odor matching and odor memory in the rat. Physiol Behav 53:795-804. CrossRef Medline

MacDonald CJ, Lepage KQ, Eden UT, Eichenbaum H (2011) Hippocampal "time cells" bridge the gap in memory for discontiguous events. Neuron 71:737-749. CrossRef Medline

Manns JR, Howard MW, Eichenbaum H (2007) Gradual changes in hippocampal activity support remember the order of events. Neuron 26: 530-540. CrossRef Medline

Markus EJ, Barnes CL, McNaughton BL, Gladden VL, Skaggs WE (1994) Spatial information content and reliability of hippocampal CA1 neurons: effects of visual input. Hippocampus 4:410-421. Medline

McCullagh P, Nelder JA (1989) Generalized linear models. Boca Raton, FL: Chapman and Hall/CRC

McNaughton BL, Battaglia FP, Jensen O, Moser EI, Moser MB (2006) Path integration and the neural basis of the 'cognitive map.' Nat Rev Neurosci 7:663-678. CrossRef Medline

Mitra P, Bokil H (2008) Observed brain dynamics. New York: Oxford UP.

Mizuseki K, Sirota A, Pastalkova E, Buzsáki G (2009) $\theta$ oscillations provide temporal windows for local circuit computation in the entorhinalhippocampal loop. Neuron 64:267-280. CrossRef Medline

Moita AP, Rosis S, Zhou Y, LeDoux JE, Blair HT (2003) Hippocampal place cells acquire location-specific response to the conditioned stimulus during auditory fear conditioning. Neuron 37:485-497. CrossRef Medline

Naya Y, Suzuki WA (2011) Integrating what and when across the primate medial temporal lobe. Science 333:773-776. CrossRef Medline

Pastalkova E, Itskov V, Amarasingham A, Buzsáki G (2008) Internally generated cell assembly sequences in the rat hippocampus. Science 321:13221327. CrossRef Medline

Robitsek JR, White J, Eichenbaum H (2013) Place cell activation predicts subsequent memory. Behav Brain Res Advance online publication. doi: 10.1016/j.bbr.2012.12.034. CrossRef

Royer S, Zemelman BV, Losonczy A, Kim J, Chance F, Magee JC, Buzsáki G (2012) Control of timing, rate and bursts of hippocampal place cells by dendritic and somatic inhibition. Nat Neurosci 15:769-775. CrossRef Medline

Skaggs WE, McNaughton BL, Gothard KM, Markus EJ (1993) An information-theoretic approach to deciphering the hippocampal code. Neural Inf Process Syst 5:1030-1037.

Song EY, Kim YB, Kim YH, Jung MW (2005) Role of active movement in place-specific firing of hippocampal neurons. Hippocampus 15:8-17. CrossRef Medline

Stapleton JR, Lavine M, Wolpert RL, Nicolelis MA, Simon SA (2006) Rapid taste responses in the gustatory cortex during licking. J Neurosci 26:41264138. CrossRef Medline

Terrazas A, Krause M, Lipa P, Gothard KM, Barnes CA, McNaughton BL (2005) Self-motion and the hippocampal spatial metric. J Neurosci 25: 8085-8096. CrossRef Medline

Tulving E (1983) Elements of episodic memory. New York: Oxford UP.

Vargha-Khadem F, Gadian DG, Watkins KE, Connelly A, Van Paesschen W, Mishkin M (1997) Differential effects of early hippocampal pathology on episodic and semantic memory. Science 277:376-380. CrossRef Medline

Wood ER, Dudchenko PA, Eichenbaum H (1999) The global record of memory in hippocampal neuronal activity. Nature 397:613-616. CrossRef Medline

Wood ER, Dudchenko PA, Robitsek RJ, Eichenbaum H (2000) Hippocampal neurons encode information about different types of memory episodes occurring in the same location. Neuron 27:623-633. CrossRef Medline 